\section{Hipatia Press}

www.hipatiapress.com

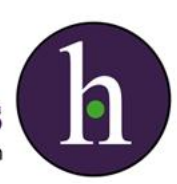

Instructions for authors, subscriptions and further details:

http://rise.hipatiapress.com

\title{
La "Roma Response" al Modelo Reproduccionista. La Educación, Nuestra Escalera para la Transformación Social
}

Jelen Amador ${ }^{1}$

1) Universidad de Barcelona, Spain

Date of publication: June $25^{\text {th }}, 2016$

Edition period: June 2016-October 2016

To cite this article: Amador, J. (2016). La "Roma Response" al Modelo Reproduccionista. La Educación, Nuestra Escalera para la Transformación Social. International Journal of Sociology of Education, 5(2), 144-163. doi: 10.17583/rise.2016.2091

To link this article: http://dx.doi.org/10.17583/rise.2016.2087

\section{PLEASE SCROLL DOWN FOR ARTICLE}

The terms and conditions of use are related to the Open Journal System and to Creative Commons Attribution License (CC-BY) 



\section{"Roma Response" to Reproductionist Model. Education, our Ladder for Social Transformation}

Jelen Amador

Universidad de Barcelona

(Received: 11 May 2016; Accepted: 25 May 2016; Published: 25 June 2016)

\section{Abstract}

The Roma Response reacts to reproductionist studies that deny the ability of education to overcome social and educational inequalities and resort to cultural difference to justify them. In this article evidences are collected showing that through school it is possible to get Roma students' academic success and to transform exclusionary contexts. That way reports as Equalitity of Educational Opportunity by James S. Coleman, drafted in 1966, are discredited. The report triggered a great impact among some following educational research. This study has led to the rejection of minority groups, like the Roma people, that have been directly affected by it and forced to social exclusion because of their supposed scientific results. In short, this paper aims to promote research, in line with the objectives of the European Commission, impact to the educational inclusion of Roma, thereby encouraging a more equal and cohesive society.

Keywords: Roma response, Roma people, education, academic success, social inclusion 


\section{La "Roma Response" al Modelo Reproduccionista. La Educación, Nuestra Escalera para la Transformación Social}

Jelen Amador

Universidad de Barcelona

(Recibido: 11 de Mayo 2016; Aceptado: 25 de Mayo 2016; Publicado: 25 Junio 2016)

\section{Resumen}

La Roma Response reacciona frente a los estudios reproduccionistas que niegan la capacidad de la educación para superar las desigualdades socioeducativas y recurren a la diferencia cultural para justificarlas. En este artículo se reúnen evidencias que demuestran que a través de la escuela es posible alcanzar el éxito académico del alumnado gitano y transformar contextos de exclusión. De esta manera, se desacreditan investigaciones como el reconocido Informe: Equalitity of Educational Opportunity de James S. Coleman, elaborado en 1966. Dicho informe desencadenó una gran repercusión entre las investigaciones educativas que le han sucedido. Esta corriente de estudios ha provocado el rechazo de grupos minoritarios que, como el pueblo gitano, se han visto directamente perjudicados y abocados a la marginación social a causa de sus resultados supuestamente científicos. En definitiva, el presente trabajo persigue promover investigaciones que, en sintonía con los objetivos de la Comisión Europea, impacten en la inclusión educativa del pueblo gitano, fomentando así una sociedad más igualitaria y cohesionada.

Palabras clave: Roma response, pueblo gitano, educación, éxito académico, inclusión social 


\section{Amador-Roma Response}

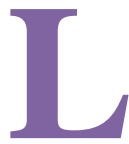

a Roma response desacredita las investigaciones educativas que desde una óptica reproduccionista anulan la capacidad de acción y de transformación de la educación. En este sentido rechaza los estudios que responsabilizan a minorías como el pueblo gitano (también conocido como Roma) de las desigualdades educativas que presentan. La Roma response reacciona así frente a esta corriente de estudios que sostiene que la escuela no es responsable de las desigualdades sociales y que tampoco puede intervenir en su superación. De lo contrario, aquí se recogen evidencias que demuestran que a través de una educación de máximos es posible vencer las barreras y dificultades académicas de cualquier tipo de alumnado, independientemente de su identidad étnica o estatus socioeconómico. En esta línea, a través de un estudio de caso, la Roma response recoge el éxito académico alcanzado por el alumnado gitano a través de la aplicación de las Actuaciones Educativas de Éxito, avaladas por la Comunidad Científica Internacional.

Seguidamente, se aportan algunos datos sobre el pueblo gitano que permiten contextualizar al colectivo objeto de estudio.

En cuanto a la dimensión de la población gitana, cabe destacar que no existen datos oficiales que calculen el número exacto de personas pertenecientes a este colectivo. Sin embargo las investigaciones apuntan que alrededor de 12 millones de personas gitanas habitan en países de Europa, América y algunas zonas de Asia y Oceanía (Sordé, Flecha \& Mircea, 2013). A nivel europeo, el pueblo gitano representa la mayor minoría étnica no migrante del continente. En este sentido, el volumen de población gitana en Europa ronda los 11 millones de personas, representando el 1,35\% de la población europea (Parekh \& Rose, 2011). En España, no se registra oficialmente la identidad étnica, por consiguiente no podemos hablar de cifras concretas. No obstante, atendiendo a las diferentes aproximaciones que ofrecen distintas investigaciones, podemos sostener que la cantidad de personas gitanas en este país oscila entre 725.000 y un millón.

A pesar de los siglos de presencia en Europa, de acuerdo con la comunidad científica, el pueblo gitano continúa siendo el grupo más rechazado y discriminado en el continente (Sordé et al., 2013). Esta realidad se remonta a tiempos pasados, en este sentido su historia viene marcada por el holocausto nazi y siglos de persecución, discriminación y exterminio (Santos, Macías, Amador \& Cortés, 2016). 
En la misma línea, la Comisión Europea, ha identificado a la comunidad gitana como el grupo con mayor riesgo a sufrir pobreza dentro del continente. Siguiendo esta tendencia, en España es el colectivo más afectado por la exclusión social severa (Gago-Cortés \& Novo-Corti, 2015). De hecho, se ven inmersos en esa exclusión uno de cada tres hogares compuestos por personas gitanas (Fundación FOESSA, 2008). Esta exclusión social, complica la participación de este colectivo en esferas de la vida social como la vida política, educativa o social. Esto revela que el pueblo gitano no es tratado de la misma manera y explica que se vea afectado por serias desigualdades materializadas en el acceso a recursos básicos como la educación, el mercado laboral, la vivienda o el sistema sanitario (Macías \& Redondo, 2012). El resultado es la generación de un círculo de pobreza y desigualdad del que tan sólo es posible salir a través de una educación de calidad que abogue por la superación de las desigualdades.

A continuación se describen las principales barreras que enfrenta el pueblo gitano en el acceso a dichos recursos básicos. Estas dificultades están estrechamente conectadas con la exclusión educativa presente en la comunidad. En este sentido, los bajos niveles de instrucción que registra complican su acceso a un mercado laboral donde las habilidades y competencias más valoradas están directamente vinculadas a la educación formal. De acuerdo con datos de 2005, solo cuatro de cada diez personas gitanas completan los estudios primarios y solo un $10 \%$ de las personas gitanas finalizan los estudios secundarios o superiores (Fundación Secretariado Gitano, 2012). En detrimento de la inserción laboral de la comunidad gitana, cada vez se valora menos el bagaje adquirido durante el desempeño de oficios irregulares, siendo cada vez más importantes para el acceso al mercado laboral las competencias acreditadas a través de titulaciones académicas.

Asimismo, entre los obstáculos que enfrenta el pueblo gitano en el acceso al empleo destaca la discriminación hacia este colectivo a lo largo de todo el proceso de contratación. Los datos ponen de manifiesto la desigualdad existente en el ámbito laboral. Así pues, en España en el año 2011 el 36.4\% de la población gitana activa estaba desempleada, mientras que en el caso de la sociedad mayoritaria el porcentaje se reducía al 20,9\% (Fundación Secretariado Gitano, 2012). Cabe destacar que la repercusión de la crisis financiera también ha sido más aguda en el caso del pueblo gitano. Puesto 


\section{Amador-Roma Response}

que este colectivo se encuentra en una situación de mayor vulnerabilidad debido, entre otras razones a los bajos niveles de instrucción que registra así como a una discriminación altamente asentada y aceptada dentro del mundo laboral.

Como estamos viendo, las barreras educativas y laborales en la comunidad gitana están estrechamente ligadas y su acumulación se convierte en un obstáculo para el acceso a otros recursos básicos como el de la vivienda. Constituye un dato muy revelador el hecho de que en España alrededor del $90 \%$ de las personas que viven en asentamientos chabolistas sean gitanas (Guglielmo, 2002). El hecho de residir en un habitáculo que no reúne las condiciones de habitabilidad y de sufrir en muchas ocasiones pobreza energética condiciona el acceso a recursos como la educación e impide la participación plena de estas personas en otras áreas de la vida social (Gago-Cortés, \& Novo-Corti, 2015). En materia de vivienda, las investigaciones denuncian la ineficiencia de algunas intervenciones y políticas públicas que promueven la segregación de la comunidad gitana. Asimismo detectan numerosos estereotipos que atribuyen al pueblo gitano una conducta incívica y delictiva. Esto acarrea un importante rechazo hacia este colectivo representando una traba en su acceso a la vivienda.

Los efectos del bajo nivel de formación dentro del pueblo gitano también se hacen palpables en su estado de salud. Las investigaciones identifican una mayor presencia de prácticas perjudiciales dentro del colectivo, como la automedicación o la falta de controles de salud periódicos. Estos hábitos no saludables están más extendidos entre los miembros de la comunidad con menos formación. En esta tónica las personas gitanas analfabetas o con bajos niveles de instrucción registran más problemas de salud que aquéllas con mayores niveles de formación (Carrasco-Garrido, López de Andrés, Hernández, Jiménez \& Jiménez, 2011). En concordancia con todo lo expuesto, esta minoría étnica registra tasas más elevadas de mortalidad infantil y de enfermedades crónicas, así como un estilo de vida menos saludable (marcado por problemas de nutrición, un mayor número de accidentes y un uso deficiente de los recursos sanitarios y de las medidas de prevención) asimismo su esperanza de vida es inferior en comparación con la sociedad mayoritaria (Sánchez, 2005; Martín-Pérez, Hernández, López de Andrés, Jiménez-Trujillo, Jiménez-García \& Carrasco-Garrido, 2015). De hecho, en Europa la esperanza de vida del pueblo gitano es 10 años inferior a 
la de la sociedad mayoritaria y esta brecha se acentúa todavía más en el caso de las mujeres gitanas (Pappa, Chatzikonstantinidou, Chalkiopoulos, Papadopoulos \& Niakas, 2015). La educación es uno de los principales recursos para la superación de esta desigualdad en materia de salud.

Para eliminar todas las barreras que afectan al pueblo gitano deviene imprescindible el desarrollo de investigaciones con impacto en la mejora de las vidas de estas personas. En esta línea, desde Europa se anima a los estados miembros a elaborar y a aplicar políticas y prácticas que contribuyan a la erradicación de los obstáculos que perpetúan la exclusión en el pueblo gitano.

En este punto, como es bien sabido, la educación juega un papel crucial, dada su capacidad para promover la inclusión y la movilidad social. Por esta razón el presente artículo identifica primeramente las barreras educativas que presenta el alumnado gitano, para después demostrar que existen evidencias que prueban que a través de la educación se ha conseguido superarlas.

La estructura del presente artículo es la siguiente, en primer lugar nos centraremos en la descripción de la situación educativa del pueblo gitano, para así conocer mejor la realidad de la que partimos. Más tarde, analizaremos la influencia de las investigaciones de orientación reproduccionista en el mantenimiento de las desigualdades educativas que afectan al alumnado gitano. Seguidamente, se recogerán las evidencias que, en contra de lo que apuntan las investigaciones reproduccionistas, demuestran que la escuela puede revertir las barreras socioeducativas y alcanzar el éxito académico de cualquier tipo de alumnado. Para ello abordaremos el estudio de caso de la escuela "La Paz". Se trata de un ejemplo de éxito con alumnado gitano en la superación de adversidades socioeducativas y cuenta con el aval de la Comunidad Científica Internacional. Finalmente presentamos las conclusiones de esta Roma Response, dónde se hace énfasis en los elementos que han sido claves en el éxito del caso de la escuela de "La Paz". De esta manera se pretende contribuir a la promoción de investigaciones que como en el caso citado tengan impacto en la mejora de la inclusión educativa de las personas gitanas. 


\section{Severas Desigualdades Educativas que Revelan la Necesidad de Investigaciones con Impacto}

Seguidamente, se aportan algunos datos cuantitativos referidos a las principales problemáticas educativas que afectan al pueblo gitano en Europa y en España. Entre las principales dificultades cabe destacar el elevado porcentaje de absentismo, fracaso escolar y analfabetismo.

Tal y como se ha expuesto anteriormente, existen serias barreras socioeconómicas que dificultan el acceso del pueblo gitano a la educación. Las investigaciones se han hecho eco de la no participación de personas gitanas en el sistema educativo, así como de la existencia de un elevado número de alumnado gitano que no asiste con regularidad a clase (Macías, \& Redondo, 2012). En la misma tónica se sitúan los resultados de la encuesta sobre la situación educativa del pueblo gitano realizada en 2011 por la European Union Agency for Fundamental Rights (FRA). Esta encuesta aborda el estudio de esta realidad en once estados europeos, entre ellos España. Señala que entre las personas gitanas de edades comprendidas en las franjas de escolarización obligatoria, una media del 14\% no asistía a clase, mientras que entre las personas no gitanas en situaciones similares el porcentaje se reducía al 3\%. En el caso de España el porcentaje de alumnado gitano absentista se incrementaba hasta el 36\% en esas edades (FRA, 2014).

Este absentismo dentro de períodos de escolarización obligatoria está estrechamente vinculado al abandono temprano de los estudios. De acuerdo con la encuesta citada, en Europa el promedio de personas gitanas de entre 18 y 24 años sin completar los estudios secundarios obligatorios alcanza el $89 \%$, mientras que en el caso de personas no gitanas en situación similar el porcentaje desciende al 38\%. Cabe destacar que esa cifra en España es más elevada que la media europea, en este sentido las personas gitanas comprendidas en esas edades y que no han completado la educación secundaria obligatoria superan el 90\% (FRA, 2014). En coherencia con este punto de partida también se aprecia una destacada infrarrepresentación del alumnado gitano en los estudios superiores (Sordé, 2006).

En la misma tónica, como ya nos hacen suponer estos datos, se advierten unos niveles de formación muy bajos dentro de la comunidad gitana. A nivel europeo, de acuerdo con la encuesta de la FRA alrededor del 20\% de las 
personas gitanas de 16 años en adelante afirma no saber leer ni escribir. En cambio, entre los participantes no gitanos el porcentaje desciende al 1\%. En España el $14 \%$ de personas gitanas de 16 años en adelante se confiesa analfabeto (FRA, 2014).

No obstante, a través del estudio longitudinal de esta realidad la European Union Agency for Fundamental Rights advierte algunas mejoras dentro del contexto académico de la comunidad gitana.

En este sentido, a nivel europeo cabe destacar el aumento de la participación del alumnado gitano en la educación formal. En esta línea, entre las generaciones más jóvenes (de entre 16-24 años) solo entre el 0\% y el $4 \%$ de las personas gitanas reconocieron no haber asistido a la escuela. Se encuentran dentro de estos porcentajes la mayoría de países abordados en la encuesta, salvo Grecia, Rumania y Francia donde la no participación en la educación formal es superior. En la misma línea, el estudio identifica un aumento en la participación de las mujeres gitanas en el sistema educativo formal (FRA, 2014).

La encuesta apunta también a un incremento en la alfabetización de las generaciones más jóvenes. En concreto, en España entre los participantes mayores de 45 años se registraba un $35 \%$ de personas analfabetas, mientras que entre los comprendidos en edades de 16 a 24 años el porcentaje se reduce al 1\% (FRA, 2014).

Si bien todavía hay mucho trabajo por hacer para mejorar la situación educativa del pueblo gitano, estos índices de mejora predicen que el cambio es posible. Estos datos esperanzadores no son arbitrarios ni casuales, sino que obedecen a todo un despliegue de investigaciones e intervenciones educativas enfocadas a la transformación y a la superación de barreras.

\section{Investigaciones Reproduccionistas que Contribuyen a Mantener la Exclusión del Alumnado Gitano}

Como ya ha quedado patente, disponemos de una gran cantidad de datos sobre la desigualdad educativa en el pueblo gitano. De la misma manera, numerosos estudios han descrito minuciosamente las problemáticas educativas que afectan al colectivo. Sin embargo, son pocas las investigaciones que se atreven a dar un paso más allá de la descripción de esta realidad para ofrecer soluciones que transformen esa situación. Incluso 


\section{Amador-Roma Response}

algunos estudios sostienen que la superación de las desigualdades a través de la educación no es factible.

Precisamente la Roma Response rebate a las investigaciones que sostienen la incapacidad de la escuela para superar las desigualdades socioeducativas, planteamiento que han mantenido estudios con un amplio reconocimiento, como es el caso del informe Equalitity of Educational Opportunity (EEO) elaborado por James S. Coleman y sus colaboradores en 1966. En este sentido, el informe aludía al contexto socioeconómico y al nivel educativo de las familias para condenar al fracaso las trayectorias académicas de las y los estudiantes pertenecientes a grupos vulnerables en riesgo de exclusión. Dicho informe alcanzó una gran influencia en investigaciones educativas posteriores. A pesar de esta buena acogida, también suscitó reacciones contrarias desde la academia, como, por ejemplo, la respuesta de autores afroamericanos a través de la conocida como Black Response (Giroux \& Flecha, 1994).

Actualmente el informe Equalitity of Educational Opportunity continúa siendo el punto de partida de muchos estudios que reafirman sus resultados. De acuerdo con los planteamientos de este informe buena parte del alumnado gitano procedente de familias con escasos recursos económicos y con bajos niveles de formación, estaría condenado a la eterna exclusión social. Puesto que no contempla la capacidad de acción de la comunidad educativa ni de los propios agentes para conseguir el éxito académico y la inclusión social.

Bajo estos parámetros reproduccionistas, la educación tendría muy poco que hacer en la superación de las desigualdades socioeducativas del alumnado gitano. La proliferación de estas convicciones ha sido muy perjudicial para el colectivo. Puesto que cuando estas ideas calan en las investigaciones y en los profesionales de la educación, el alumnado gitano pasa a ser un problema. Por consiguiente, en base a la idea de que la educación no tiene nada qué hacer, buena parte de las prácticas educativas se ha dirigido a apartar y a entretener al alumnado gitano. Las investigaciones denuncian que en Europa todavía se segrega a niñas y niños gitanos que a menudo son automáticamente concentrados en centros de educación especial, a pesar de que no se dan las circunstancias necesarias para justificarlo (Sordé, 2006; O’Nions, 2010). En la misma línea, la encuesta ya citada de FRA apunta que uno de cada diez niños gitanos encuestados había 
asistido a una escuela especial o aula destinada principalmente a gitanos (FRA, 2014). Uno de los propósitos que se oculta detrás de estas prácticas es evitar que el alumnado gitano entorpezca el proceso de aprendizaje del alumnado exento de desigualdades socioeducativas y con expectativas de éxito académico de acuerdo con la perspectiva reproduccionista.

Asimismo, estas ideas reproduccionistas justifican que en muchas ocasiones se imparta una educación de mínimos cuando se trata de alumnado gitano. De acuerdo con estas ideas deterministas, pierde sentido aspirar al éxito académico de este colectivo y resulta más rentable apartarlos y reducir el nivel de su educación para facilitarles el seguimiento de los contenidos. Entre los argumentos más recurrentes para la justificación de este tipo de prácticas discriminatorias destacan la falta de dominio del idioma oficial, en aquéllos países donde la comunidad gitana mantiene y utiliza el romanó, un nivel de formación insuficiente o las conductas absentistas (Kyuchukov, 2000).

El papel del pueblo gitano en este contexto de exclusión educativa es muy limitado. Con frecuencia, las familias gitanas no tienen la oportunidad de participar a la hora de decidir si se aparta del grupo al alumnado gitano o si se rebaja el nivel de la educación que se le ofrece. En este sentido, a menudo se entiende que las familias gitanas no pueden realizar aportaciones al debate educativo. Y son los profesionales de la educación quienes, como conocedores expertos de la situación y dada la posición que ostentan, deciden qué educación es la que conviene al alumnado gitano. Sin embargo, las familias gitanas denuncian que cuando sus hijas e hijos son concentrados en espacios educativos donde se les ofrece una educación de peor calidad, como en el caso de las escuelas gueto, no adquieren las habilidades necesarias ni se forman de acuerdo con la diversidad presente en la sociedad real (Igarashi, 2005).

La no participación unida a la no representación de los intereses del pueblo gitano en los procesos educativos de decisión, dan lugar a que los estereotipos sobre esta minoría étnica estén muy presentes y no puedan ser rebatidos a través del diálogo entre los profesionales de la educación y los agentes la comunidad gitana.

De la misma manera, cuando no se tiene en cuenta al colectivo en el desarrollo de las investigaciones, es habitual que desde una perspectiva relativista se alcancen interpretaciones como la que realiza la autora Okely 


\section{Amador-Roma Response}

en referencia al elevado porcentaje de analfabetismo en el pueblo gitano. La autora sostiene que, lejos de ser un hándicap, este analfabetismo es una muestra de la libertad que caracteriza a la comunidad gitana (Touraine, Flecha, \& Wieviorka, 2004).

Los resultados de toda esta corriente de estudios reproduccionistas han alcanzado a una parte de la comunidad científica, influyendo en el diseño de muchas de las prácticas educativas que hoy en día se aplican al alumnado gitano. Uno de los estereotipos más difundidos a través de las investigaciones que parten de esta perspectiva, responde a la idea de que las familias gitanas no están interesadas en la educación y que los elevados índices de absentismo o de fracaso escolar obedecen a ese desinterés (Flecha \& Soler, 2013). Atendiendo a este desinterés por la educación, el punto de partida se convierte en la necesidad de concienciar a las familias de su importancia y motivar al alumnado gitano a asistir y a participar en el sistema educativo. Bajo este argumento se justifican prácticas discriminatorias como el impartir actividades de tipo lúdico o manual para el alumnado gitano mientras que el resto del grupo se está formando en conocimientos instrumentales.

Otro de los estereotipos más extendidos es que el respeto a la libertad del pueblo gitano justifica que se le exima de cumplir con algunas normas de funcionamiento del centro escolar como el horario de inicio, fomentando así la falta de disciplina.

Es evidente que cuando de acuerdo con la perspectiva reproduccionista, el éxito académico del alumnado gitano no puede ser la meta de los planteamientos educativos, emergen prácticas que, como hemos visto apuestan por la rebaja de la educación, así como interpretaciones que incluso justifican el analfabetismo en esta minoría étnica. Beben de todas estas ideas, prácticas educativas como la adaptación curricular o los grupos homogéneos. Para sostener estos planteamientos a menudo se recurre a un compromiso con la defensa de la diferencia cultural. Sin embargo, estas ideas son muy perjudiciales para el pueblo gitano. En primer lugar, pierden de vista el objetivo de la igualdad, promoviendo a través de la rebaja de la calidad de la educación el fracaso educativo del alumnado gitano, prolongando así la exclusión social. En segundo lugar, promueven una imagen de la identidad gitana basada en estereotipos sin representar los valores del propio colectivo. En base a estas razones, las investigaciones que 
sostienen estos argumentos y las prácticas que beben de éstas ideas han sido abiertamente rechazadas por el pueblo gitano (Aiello, Mondejar \& Pulido, 2013).

Entre los efectos colaterales de estas prácticas de base reproduccionista advertimos la promoción de la idea de inferioridad de las personas gitanas, el fomento de la intolerancia, así como de la discriminación del alumnado gitano (O’Nions, 2010). A la misma vez revela una ausencia de expectativas, refuerza los estereotipos, daña la autoestima de las y los estudiantes gitanos y provoca la pérdida de sentido de la educación.

De la misma manera, estas prácticas de base reproduccionista eliminan cualquier posibilidad de transformación y después responsabilizan al alumnado gitano del fracaso y el abandono escolar que acaban experimentando como resultado de la aplicación de prácticas que ya en su diseño descartaban cualquier posibilidad de éxito académico.

A estas prácticas discriminatorias en materia de educación se suman barreras educativas que afectan al pueblo gitano. En esta línea, a la ausencia de referentes gitanos con estudios superiores se une la soledad que a medida que avanzan los cursos experimentan las y los estudiantes gitanos que continúan con su formación, especialmente en el ámbito de los estudios superiores donde la infrarrepresentación del colectivo es mayor ( $\mathrm{O}^{\text {'Nions, }}$ 2010). A esto se añade la invisibilidad de la identidad gitana en el curriculum y entre el personal docente. A todo esto hay que sumar las bajas expectativas con frecuencia reforzadas desde la comunidad educativa. La acumulación de todas estas barreras a menudo acaba frustrando la trayectoria académica del alumnado gitano.

\section{Roma Response: La Escuela de "La Paz", de Reproducir a Transformar las Desigualdades del Pueblo Gitano}

Esta Roma response en contra de la visión reproduccionista, analiza el estudio de caso de la escuela "La Paz" en busca de evidencias que prueben que el éxito educativo del alumnado gitano puede ser una realidad. En primer lugar, presentamos las características del contexto socioeconómico del barrio de La Milagrosa, donde se ubica esta escuela. Asimismo, abordaremos los cambios que se vivieron en la escuela de "La Paz" en el curso 2006-2007. También indagaremos acerca de los elementos que 


\section{Amador-Roma Response}

provocaron que la escuela pasara de ser una escuela abocada al fracaso a ser una escuela con éxito académico.

La escuela "La Paz" se ubica en el barrio de La Milagrosa, localizado a las afueras de la ciudad de Albacete (España). Este barrio surge en la década de los 70 con el objetivo de erradicar el chabolismo a través del realojo de familias, en su mayoría gitanas. Se trata de una barriada bastante aislada con una escasa cobertura de transporte público y con resistencias entre los taxistas para acceder al barrio.

El total de habitantes en La Milagrosa según datos publicados en 2010, alcanzaba los 2.385, representando el 1,5\% de la población total de Albacete (Girbés, Amador \& Macías, 2015). El barrio destaca por tener una población muy joven. Los menores de 14 años constituyen el 33,68\% del total de habitantes de La Milagrosa (aproximadamente el doble de lo que supone este grupo de edad si nos referimos a la población total de la ciudad) (Brown, González \& Pascual, 2013). Asimismo, destaca el volumen de población gitana que representa el $90 \%$ de los habitantes de La Milagrosa (Flecha \& Soler, 2013).

La Milagrosa, conocida también como el barrio de "Las 600", es uno de los barrios más desfavorecidos de Europa. Registra unos niveles de formación muy bajos, de hecho, el porcentaje de personas de más de 16 años que son analfabetas o que no han completado los estudios básicos es del 77,4\% (Girbés et al., 2015). La situación laboral es muy precaria y viene marcada por elevados índices de desempleo, salarios muy bajos y por el ejercicio de actividades informales como la venta ambulante o la recogida de residuos metálicos. La importante escasez de recursos económicos lleva aparejado un elevado índice de dependencia entre las y los vecinos del barrio. En cuanto a la convivencia en esta localidad, se registraba un alto nivel de conflictividad ocasionado por problemáticas como el tráfico y el consumo de drogas, el elevado porcentaje de personas en prisión y la inseguridad en el barrio. Esta realidad estaba estrechamente conectada al contexto de pobreza y marginación y complicaba la convivencia en esta zona y en espacios públicos como la escuela (Girbés et al., 2015).

La escuela era un reflejo de ese contexto de conflictividad y exclusión social. Los conflictos en el centro escolar eran frecuentes y su trayectoria venía marcada por el elevado nivel de fracaso escolar y de absentismo (alrededor de un 30\%). Esta secuencia de problemáticas acabó provocando 
el cierre del centro escolar en el curso 2005-2006.

La gravedad de la situación representó un punto de inflexión. Frente a esta realidad, las autoridades educativas decidieron reemprender un nuevo proyecto educativo creando un nuevo colegio en el mismo espacio, pero con una clara orientación hacia el cambio social y la transformación del contexto.

Se emprende ese nuevo proyecto educativo y la nueva escuela "La Paz" abre sus puertas en el curso 2006-2007. La escuela se componía mayoritariamente de familias en situación de riesgo social y con ingresos muy bajos. El alumnado gitano alcanzaba aproximadamente el 50\% del total de los estudiantes, la presencia de alumnado migrante era menor y rondaba el $7,1 \%$. Este alumnado migrante procedía principalmente de África, Sud América y el Este de Europa (INCLUD-ED, 2010).

La base del nuevo proyecto educativo consistía en la aplicación de las Actuaciones Educativas de Éxito (AEE), identificadas a través del proyecto de investigación INCLUD-ED. Strategies for inclusion and social cohesion in Europe from education (2006-2011), enmarcado en el VI Programa Marco de investigación de la Comisión Europea. El impacto educativo alcanzado por el proyecto INCLUD-ED le ha llevado a ser la única investigación en Ciencias Sociales y Humanidades incluida en la lista de las 10 "success stories" de la Comisión Europea.

Las AEE, como recoge el monográfico en el que se integra el presente artículo, consisten en la explotación de todos los recursos disponibles en la escuela y la comunidad para mejorar el rendimiento académico del alumnado. Son el resultado de un exhaustivo análisis de los sistemas educativos, teorías y prácticas, especialmente de las acciones de éxito que han conseguido resultados excelentes en el alumnado procedente de familias con un bajo nivel socioeconómico (Flecha \& Soler, 2013). La aplicación de las Actuaciones Educativas de Éxito ha mejorado los resultados académicos de muchos estudiantes, incrementando sus oportunidades y reduciendo las desigualdades socioeconómicas. Las AEE han demostrado conseguir los mejores resultados académicos con cualquier perfil de alumnado y en cualquier contexto. Esto obedece a que las AEE han probado que son universales y transferibles.

En este sentido, con la aplicación de las AEE la escuela de "La Paz" experimentó un cambio que se hizo palpable más allá del propio centro. 


\section{Amador-Roma Response}

Estas actuaciones involucran a toda la comunidad educativa y prevén la participación de las familias en actividades académicas como los grupos interactivos o la formación de familiares, así como en los procesos de decisión. De esta manera se estableció un diálogo igualitario entre el personal docente, formado en las bases de las AEE, y las familias gitanas del centro. Esta forma de participación invirtió la situación conflictiva que hasta entonces había enfrentado al profesorado y a las familias. Así, se generó un ambiente de colaboración y solidaridad que puso fin al malestar que imperaba anteriormente y que creó un sentimiento de pertenencia entre las familias gitanas que anteriormente no sentían la escuela como propia, sino como una institución alejada que no contribuía a superar desigualdades.

En cuanto al impacto en los resultados académicos, de acuerdo con los test del Departamento de Educación, el alumnado de la escuela "La Paz" mejoró considerablemente su rendimiento educativo. De hecho, de acuerdo con estas pruebas en el curso 2007-2008 los alumnos de ocho años aumentaron su puntuación respecto al curso anterior, pasando de 1 a 2,5 (sobre 5) puntos en Lengua. Además, esta mejora se mantuvo en los siguientes cursos. Asimismo, los alumnos de 9 años aumentaron sus resultados en matemáticas respecto al curso anterior pasando de 1 a 3 puntos (sobre 5). Estos son sólo algunos ejemplos del incremento de la puntuación en diferentes materias incluidas en las pruebas del Departamento de Educación (Flecha \& Soler, 2013).

Todas estas mejoras devolvieron el sentido de la educación para las familias y el alumnado gitano de la escuela de "La Paz". En este sentido, la percepción de los alumnos va en la misma línea que los datos oficiales del Departamento de Educación, puesto que el alumnado gitano también era consciente de la mejora de su aprendizaje. Este planteamiento de una educación de máximos para todos aumentó las expectativas del alumnado y logró superar problemáticas como el absentismo. Hasta el punto de que si en el curso 2006-2007 el porcentaje de absentismo en "La Paz" era del 30\%, en el curso 2007-2008 se redujo al 10\%, mientras que en el curso 2009-2010 pasó a ser algo ocasional (Flecha \& Soler, 2013).

Por otro lado, la participación de familiares y personas gitanas en la escuela superó los problemas de invisibilidad e infrarrepresentación de la identidad gitana en este espacio, generando referentes gitanos para los alumnos y alumnas pertenecientes a esta minoría étnica. Esto empodera al 
alumnado gitano y aumenta su horizonte de expectativas académicas, rompiendo con el dilema al que muchos se ven arrastrados cuando tienen que escoger si continuar formándose en un sistema educativo donde la identidad gitana está invisibilizada (Sordé, 2006).

Esta mejoría no pasó desapercibida y fomentó un aumento en la demanda de matrículas en el centro. Esto es una evidencia de cómo a raíz de la aplicación de las Actuaciones Educativas de Éxito este centro educativo pasa de ser una escuela abocada fracaso y rechazada por las familias a ser una escuela de éxito con mucha demanda. Asimismo, cabe destacar que esta escuela ha sido premiada por el cambio social que ha conseguido. El caso de la escuela "La Paz" constituye una muestra de que la identidad étnica del alumnado no tiene por qué ser un problema y a la misma vez corrobora la capacidad de la educación para superar barreras en beneficio de la igualdad y la inclusión social.

\section{Conclusiones}

Esta Roma response sostiene que la educación sí que contribuye a transformar la realidad y a superar las desigualdades socioeducativas.

Tal y como demuestra el estudio de caso de la escuela "La Paz", las prácticas educativas basadas en evidencias y avaladas por la Comunidad Científica Internacional como las AEE superan la exclusión educativa en cualquier tipo de alumnado incluido el alumnado gitano. Identificar esta garantía científica de impacto es imprescindible. La implementación de prácticas educativas basadas en opiniones, ocurrencias o estereotipos ha sido lamentablemente muy habitual en el caso del alumnado gitano. Agravando la situación del colectivo, puesto que ha perpetuado las desigualdades y a menudo ha acabado haciendo responsable al pueblo gitano de ese fracaso.

Como hemos visto, en el proceso orientado a alcanzar el éxito académico del alumnado gitano ha sido clave la incorporación de la voz de la propia comunidad. Este planteamiento es muy enriquecedor, puesto que aúna el conocimiento científico existente aportado por los investigadores y la realidad empírica que facilita el colectivo estudiado. Como resultado de esta simbiosis se alcanza un conocimiento científico nuevo que goza de mayor representatividad y de mayor impacto. Además, esta dinámica da respuesta a las reivindicaciones del pueblo gitano que denuncia su escasa representación 


\section{Amador-Roma Response}

en las investigaciones, demandando una mayor participación en las mismas: "las gitanas y gitanos hemos sido excluidos hasta de los discursos sobre la exclusión. Si una parte de la comunidad científica internacional nos ha ignorado hasta ahora, otra nos ha estudiado excluyendo nuestras voces de sus análisis y cayendo en estereotipos racistas" (Touraine et al., 2004, p.8).

Entre los beneficios de la participación de las familias gitanas en la escuela y en los procesos de decisión, cabe destacar la mejora en la resolución de conflictos así como la construcción de un ambiente de colaboración. A través de esta participación las familias se involucran en una escuela donde se les tiene en cuenta y que sienten como propia. Esta participación también contribuye a mejorar el rendimiento del alumnado y a solventar barreras como la invisibilidad de la identidad gitana en los centros educativos o la falta de referentes gitanos.

En definitiva, este estudio desmonta a través de evidencias los estereotipos que responsabilizan a minorías como el pueblo gitano del fracaso escolar. Puesto que como ha quedado demostrado, existen actuaciones educativas que han conseguido los mejores resultados académicos independientemente del estatus, género, etnia o cualquier otra característica del alumnado. Por otro lado, la identificación de los elementos que han sido cruciales en el éxito alcanzado por la escuela de "La Paz" representan una contribución para un mayor impacto de las investigaciones en la inclusión socioeducativa de colectivos vulnerables como el pueblo gitano.

\section{Referencias}

Aiello, E., Mondejar, E., \& Pulido, M. Á. (2013). Communicative Methodology of Research and Recognition of the Roma People. International Review of Qualitative Research, 6(2), 254-265. doi: 10.1525/irqr.2013.6.2.254

Brown, M., González, A. G., \& Pascual, A. M. (2013). Procesos dialógicos de planificación de los servicios sociales: el proceso de cambio en los barrios de La Milagrosa y La Estrella (Albacete). Scripta Nova: revista electrónica de geografía y ciencias sociales, 17, 6. 
Carrasco-Garrido, P., De Andrés, A. L., Barrera, V. H., Jiménez-Trujillo, I., \& Jiménez-García, R. (2011). Health status of Roma women in Spain. The European Journal of Public Health, 21(6), 793-798. doi:

10.1093/eurpub/ckq153

European Union Agency for Fundamental Rights. (2014). Education: The situation of Roma in 11 EU Member States. Luxembourg:

Publications Office of the European Union. doi:10.2811/53012

Flecha, R., \& Soler, M. (2013). Turning difficulties into possibilities:

Engaging Roma families and students in school through dialogic learning. Cambridge Journal of Education, 43(4), 451-465. doi: 10.1080/0305764X.2013.819068

Fundación Foessa. (2008). VI Informe sobre exclusión y desarrollo social en España 2008 (Vol. 11). Cáritas Española.

FUNDACIÓ, P. T. (2005). Estudi sobre la població gitana de Catalunya: informe final. Dir: Montse Sánchez Aroca-Barcelona: Generalitat de Catalunya: Departament de Benestar i Familia.

Fundación Secretariado Gitano. (2012). Población gitana, empleo e inclusión social. Un estudio comparado: Población gitana española y del Este de Europa.

Gago-Cortés, C., \& Novo-Corti, I. (2015). Políticas inclusivas de vivienda a través de la actuación municipal en la erradicación del chabolismo: El realojo del poblado de Culleredo (Galicia, España). Gestión y política pública, 24(1), 131-167.

Girbés, S., Amador, J., \& Macías, F. (2015). La transformación de la escuela "La Paz". Un modelo dialógico de intervención social para la inclusión del pueblo Roma. Modelos e projetos de inclusão social, 16-31.

Giroux, H. A., \& Flecha, R. (1994). Igualdad educativa y diferencia cultural. Barcelona: El Roure.

Guglielmo, R. (2002). Seguimiento de la protección de las minorías en la Unión Europea: La situación de los roma/gitanos en España. Budapest: Open Society Institute,

Igarashi, K. (2005). Support programmes for Roma children: do they help or promote exclusion?. Intercultural education, 16(5), 443-452. doi:10.1080/14675980500378508 


\section{Amador-Roma Response}

INCLUD-ED. Strategies for inclusion and social cohesion in Europe from education. (2006-2011). $6^{\text {th }}$ Framework Programme. Citizens and Governance in a Knowledge-based Society. CIT4-CT-2006-028603. Directorate General for Research, European Commission.

INCLUD-ED. (2010). Working papers: Case studies of local projects in Europe (4th Round). Project 6/ WP22: Case analysis of local projects working towards social cohesion. Strategies for inclusion and social cohesion in Europe from education. Directorate General for Research, European Commission.

Kyuchukov, H. (2000). Transformative education for Roma (Gypsy) children: an insider's view. Intercultural education, 11(3), 273-280. doi:10.1080/14675980020002420

Macías, F., \& Redondo, G. (2012). Pueblo gitano, género y educación: investigar para excluir o investigar para transformar. International Journal of Sociology of Education, 1(1), 71-92. doi: 10.4471/rise.2012.04

Martín-Pérez, M., Barrera, V. H., de Andrés, A. L., Jiménez-Trujillo, I., Jiménez-García, R., \& Carrasco-Garrido, P. (2015). Predictors of medication use in the Roma population in Spain: a population-based national study. Public health, 129(5), 453-459. doi:

10.1016/j.puhe.2015.01.028

O'Nions, H. (2010). Different and unequal: the educational segregation of Roma pupils in Europe. Intercultural Education, 21(1), 1-13. doi:10.1080/14675980903491833

Pappa, E., Chatzikonstantinidou, S., Chalkiopoulos, G., Papadopoulos, A., \& Niakas, D. (2015). Health-Related Quality of Life of the Roma in Greece: The Role of Socio-Economic Characteristics and Housing Conditions. International journal of environmental research and public health, 12(6), 6669-6681. doi:10.3390/ijerph120606669

Parekh, N., \& Rose, T. (2011). Health inequalities of the Roma in Europe: a literature review. Central European journal of public health, 19(3), 139.

Santos, T., Macías, F., Amador, J., \& Cortés, D. (2016). Samudaripen, el Genocidio Gitano: ¿Volveremos a Repetir la Historia?. Scientific Journal on Intercultural Studies, 2(1), 2-29. doi:10.17583/recei.2016.1897 
Sordé, T. (2006). Les reivindicacions educatives de la dona gitana. Cabrera de Mar: Edicions Galerada/Institut Català de les Dones.

Sordé, T., Flecha, R., \& Mircea, T. (2013). El pueblo gitano: una identidad global sin territorio. Scripta Nova: Revista electrónica de geografía y ciencias sociales, 17, 3 .

Touraine, A., Flecha, R., \& Wieviorka, M. (2004). Conocimiento e identidad. Barcelona: Editorial El Roure.

Jelen Amador is PhD Student at Department of Sociology at University of Barcelona.

Contact Address: Direct correspondence to Jelen Amador, Department of Sociology, University of Barcelona, Diagonal Nord, Facultat d'Economia i Empresa (Edifici 690) 08034 Barcelona.

E-mail: jelen.amador@ub.edu 